\title{
Analysis of online Shopping Fakes from the Perspective of Information Asymmetry \\ Kunzhuo Song
}

School of Economics and Management, Beijing Jiaotong University, P.R China, 100044

15120527@bjtu.edu.cn

Keywords: Online shopping fakes, Information asymmetry, Lowercase letters.

\begin{abstract}
Nowadays, the online shopping market is very influential.To bring consumers convenient at the same time, there are many hidden dangers, from come out so far, has been a problem of fake online shopping market but around the ridge, like a raging fire double eleven, every year to refresh a new record, but the fake problem still exists. This paper from the information asymmetry theory as a starting point, the first to explain the asymmetric information theory, the method of using the signaling and screening to solve the adverse selection problem caused by asymmetric information, the application of the theory of asymmetric information in e-commerce market, also made a detailed description. In this paper, the game theory model is used to explain the phenomenon of fake goods in the online shopping market, and based on this model, some suggestions are put forward to solve the fake problem in the online shopping market.
\end{abstract}

\section{Introduction}

2016 Tmall double eleven is very hot, creating a sales volume of 24 hours of 90 billion. But we can not buy cheap and right goods, it may be fake. The main reason for the fake is information asymmetry. First of all, the information device in Taobao mainly includes the following three items, namely: credit evaluation system, interactive communication platform and payment platform. Relative to the consumers, sellers know more about the quality of their products, and through years of sales data analysis and experience, they also understand consumer psychology and possible disputes, to facilitate access to the use of existing Tmall and Taobao policy, so it has more advantages in pricing, products and other aspects. Specifically, the seller can determine the location of the consumer through the analysis of his ability to identify the fake goods, and decided to send what kind of goods. If it is in a small city, then the recipient's ability to identify, especially the ability to identify the big name products may be poor, then the possibility of sending fake identification is lower, and higher income. Again, through the analysis of the past history of consumer consumption, if consumers rarely buy price more than 100-200 yuan of products, and when the double eleven is to buy a single brand, then he is likely to be a famous brand but do not understand before sale of disposable consumer customers, then send him the product is fake the probability is relatively high. At the same time, due to the use of a number of marketing tools, confusing information, making consumers more difficult to identify. For example, back now, find someone to brush comments, etc.. The consumer information channel is relatively narrow, in the unfavorable position of information. Consumers often only gets the standard from the shop price, pictures, product description information, obtain the subjective information from other people's comments, buyers show from the shops, the reputation of Star shop, and before the introduction shows that the three kinds of information are actually from the store can control or influence, thus. The consumer is in the disadvantage of information. Moreover, due to the return of online shopping is more trouble, many people will not choose to return or bad reviews, which also makes a lot of people can not get feedback from the evaluation. Some of the stores because the offer is too low, fake has become a means of profit, so the shop business is profitable to sell fake, fake will not disappear. 


\section{Theory and application of information asymmetry}

2.1 Theoretical explanation. The asymmetric information theory research in the transaction, than the other party to hold more information, the two sides in the transaction is not equal, which leads to market failure. Akerlof (1970) believes that due to asymmetric information, resulting in the quality of uncertainty, resulting in shrinking transactions. A study on the quality uncertainty of the used car market in the United states. The quality of second-hand car "cherry" and "lemon" cheap second-hand car in the second-hand car market, because there are many factors that are not known, as the former owner of the driving style, the main parts of automobile maintenance records and historical accidents hidden inside the car can not see. Buyers don't know what to buy the car in the end is "cherry" or "lemon", so the best guess for the average quality of the buyer, he is willing to pay an average price. Only means that the quality of the used car cherry can only get the average price, and lower than its actual value, cherry sellers feel unfair transactions, it will withdraw from the used car market. Repeat the cycle, the medium will remove the second-hand car market, the average quality of second-hand cars continue to decline, finally only a lot of "lemon" in the market, and eventually lead to the shrinking market.

Signal and filter can be used to solve the adverse selection problem caused by information asymmetry. Spence (1973) first put forward the theory of signal, he believes that in the case of asymmetric information, one party to the other party to transmit information, to solve the information asymmetry. Spence to work as an example to illustrate the role of the signal. If the employer needs to find a good learning staff, and all potential employees have claimed to be good at learning, and the learning ability only they know, this has brought about the employee's learning ability information uncertainty. It is generally accepted that college graduates are more capable of learning than others, so that college graduates can be regarded as signals of learning ability. Therefore, potential employees with college graduates will pass this information on to employers as evidence of their ability to learn. Rothschild and Stiglitz (1976) proposed the screening mechanism to solve the problem of information asymmetry. A party who is in a weak position can induce the dominant party to disclose more information.

2.2 Application theory. Compared with the traditional used car market, the quality of the goods in the e-commerce market, there is a huge uncertainty, the seller has more information about the quality of the product than the buyer, the seller is in the information disadvantage of the party. Because of the limited information available to buyers, it is impossible to distinguish between the goods offered by different sellers, so the goods are homogeneous in their eyes. So in theory, in the e-commerce market, more and more high-quality goods will gradually withdraw from the market, and finally only the low quality of the goods, causing the market to shrink. However, we are worried that things have not been found, the e-commerce market has been telling the development. Therefore, we have reason to believe that, while the information asymmetry theory plays a role, there is a mechanism that can effectively curb the "lemon" in the e-commerce market".

As a kind of effective signal mechanism, the buyer can get more information about product quality and reduce the uncertainty of product quality. The sellers of goods of high quality can gain additional benefits, and can continue to stay in the e-commerce market, so they will not be squeezed out of the market. We believe that the credibility of the feedback mechanism at least partially prevent the evolution of the e-commerce market to lemon market to ensure the long-term development of e-commerce market power.

\section{Analysis based on game theory model}

3.1 Before the constraint mechanism is introduced. Enterprises to enter the e-commerce market cost for $\mathrm{C}$, namely platform construction costs and management costs; high price customers pay for products and services, pay low prices for products and services; $(\mathrm{i}=\mathrm{h}, \mathrm{l})$ on behalf of the customer through the purchase of product benefits, which represent income to buy high priced products that represents the purchase low price earnings; as B enterprise must spend some cost of camouflage can 
hope to deceive the buyer, the cost for camouflage. The benefits of the seller and the buyer can be expressed in the form of extension in figure 1:

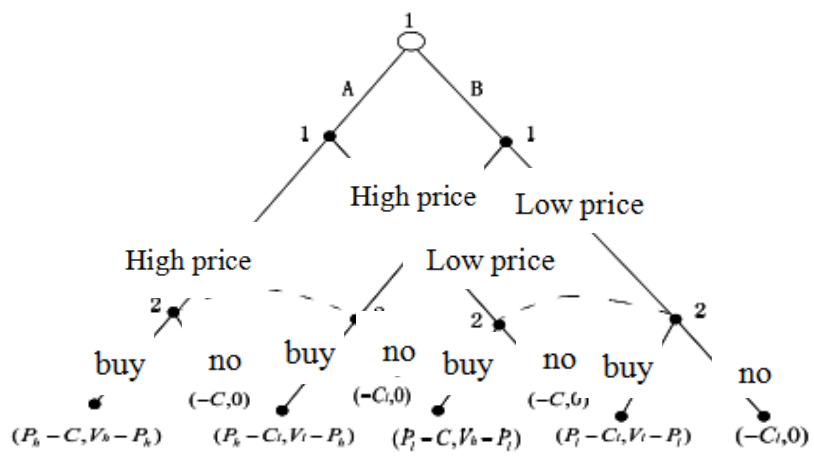

Fig.1 The game model of asymmetric information market

From the analysis of the behavior, regardless of the A type B type to the seller or seller has sold their products with complete information, but the cost of goods sold by the seller of A type is higher than the cost of goods sold by the seller of B type, not willing to sell at a low price or even at a loss. Therefore, for the A type of seller, its dominant strategy is "high price", and the dominant strategy of B type seller is "low price"".

From the analysis of the buyer's behavior: the buyer and the seller of the type A to reach a deal, both sides can get a positive return. The buyer and the seller of the type B will suffer losses. Since the seller has private information about the company and the product, and the buyer does not know, the buyer assumes that the seller is A, B two types of probability of existence are $\mathrm{p}(\mathrm{g} \mid \mathrm{s}), \mathrm{P}(\mathrm{b} \mid \mathrm{s}) \mathrm{P}$ $(\mathrm{g} \mid \mathrm{s})+\mathrm{p}(\mathrm{b} \mid \mathrm{s})=1$. As a rational game in which the buyer is unable to obtain more information about the seller, it is assumed that $\mathrm{P}(\mathrm{g} \mid \mathrm{s})=\mathrm{p}(\mathrm{b} \mid \mathrm{s})=0.5$. Thus, in both revenue models set under the condition of buyer's choice of "high price" strategy: expected return; buyer's choice of "low price" strategy: the expected return is obviously, the buyer's choice of "low price" strategy of the expected return is greater than the buyer's choice of "high price" strategy of the expected return, so the buyer's optimal strategy is "low". Under this equilibrium strategy, the buyer is more inclined to trade with the seller of B type. If this situation continues, it will lead to bad money drives out good money phenomenon, the credibility of the seller was forced to leave the market, the transaction rate of online transactions will become very low. Pareto optimal supply is difficult to achieve, the market will fail, and ultimately the formation of e-commerce lemon market results.

3.2 After introducing the constraint mechanism. By introducing the punishment mechanism and credit evaluation mechanism and restraint mechanism, while keeping the hypothesis model in a constant, and adding a new variable t, adjusted to the seller's benefit, to build a new game as shown in figure 2.

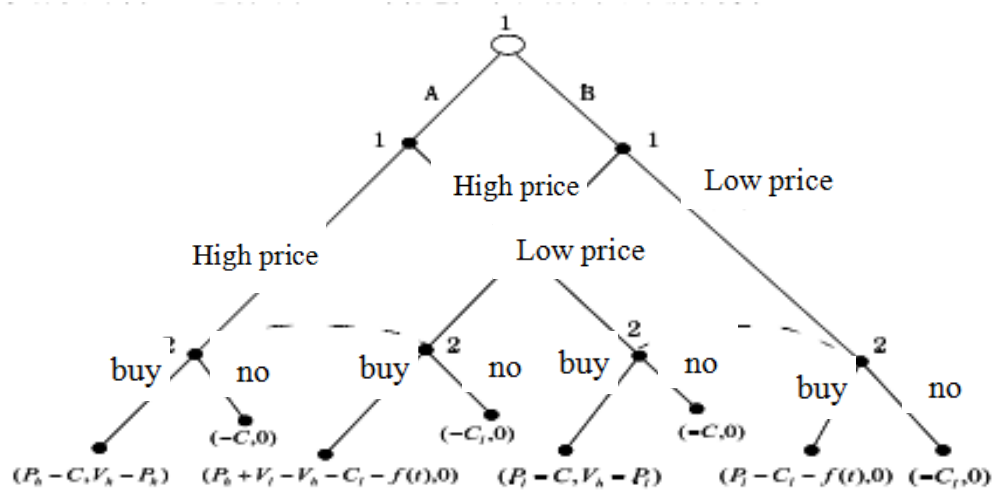

Fig.2 The game model based on constraint mechanism 
In this model, it is used to evaluate the seller's reputation. That is, in the transaction once the seller provides false products or poor service, $t$ increased by 1 . Once $t$ becomes positive for the seller will be reduced to 0 , rational buyers will not buy their products. The credit value of the independent variable $\mathrm{t}, f(t)=\left\{\begin{array}{l}+\infty, t \neq 0 \\ 0, t=0\end{array}\right.$

After the punishment mechanism and reputation mechanism into the online retail market, if the seller to the buyer to provide quality products, all losses caused by the buyer to buy inferior products will be compensated, and the seller is reduced to 0. Such a system is clearly an expensive undertaking. For the buyer, the punishment mechanism to ensure that he buy the products and services you also do not have what loss, therefore his dominant strategy is to (buy); in addition, for A enterprises, because it is to provide good quality products and services, and the dominant strategy is to buy, so do not worry about not sell or worry the buyer's claim. For B enterprises, the introduction of punishment mechanism and reputation mechanism to increase the cost of speculation. The choice of negative income, provide inferior products will get the optimal strategy for enterprises is to provide quality products for the benefit of P-C.

In this way we can get the perfect Bias equilibrium of the new game: a class of enterprises to provide quality products and services, buyers choose to buy, at this time the buyer's judgment is $\mathrm{p}$ $(\mathrm{g} \mid \mathrm{s})=1, \mathrm{P}(\mathrm{b} \mid \mathrm{s})=0$.

\section{Conclusion}

In the second model, through the introduction of the punishment mechanism and reputation evaluation mechanism, the buyer in the transaction due to asymmetric information and all the risks borne by the seller to provide poor quality products and services. It can be seen that the speculative cost of the seller to rely on the advantage of information to carry out the moral hazard behavior is enormous. On the one hand, the buyer can according to the quality of the seller credit evaluation identification, on the other hand, the market punishment mechanism enable the buyer not because the information disadvantage and damage their own interests, so as to reduce the chance of adverse selection, and will also improve the cost of cheating buyer. Therefore, the reputation mechanism can distinguish the authenticity of the supplier information, but also provides a guarantee for the integrity of suppliers.

\section{References}

[1] Zhao Ruitao. Empirical Study on the influence of seller's reputation on sales in online trading [D]. Jilin: Jilin University, 2011:8-16, 22-27.

[2] Xie Aiping, Fu Ping. Game Analysis of asymmetric information in online retail market [J]. software engineer, 2014 (5): 58-60.

[3] Zhang Yi. Taobao.com in information and efficiency of [D]. Beijing: Minzu University of China, 2013:26-44.

[4] Zhang Ping. Analysis and Countermeasures of consumer research in online shopping era [N]. Journal of Shandong TV University. 2012:4.

[5] Yuan Xin, Hu Tian Xin, Li Qingchuan,.. A study on the risk of online shopping [J]. Oriental enterprise culture, 2012.9. 